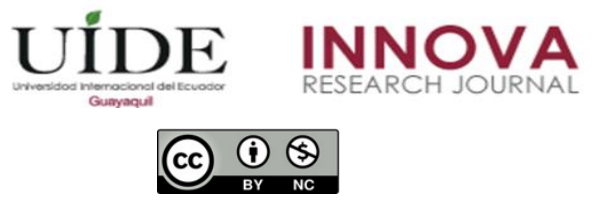

INNOVA Research Journal, ISSN 2477-9024

(Septiembre-Diciembre 2020). Vol. 5, No.3.1 pp. 1-14

DOI: https://doi.org/10.33890/innova.v5.n3.1.2020.1485

URL: http://revistas.uide.edu.ec/index.php/innova/index

Correo: innova@uide.edu.ec

\title{
Formación interdisciplinar STEAM + A: vocaciones por género y resultados de la experiencia
}

\section{Interdisciplinary training STEAM + A: vocations by gender results of the experience}

\author{
Albenis Cortés Rincón \\ iD http://orcid.org/0000-0001-7172-5699 \\ Víctor Nicolás Álvarez Monroy \\ (D) https://orcid.org/0000-0002-7070-3698 \\ Fundación Universitaria Compensar, Colombia
}

Autor para correspondencia: albeniscortes@unipanamericana.edu.co; vnalvarezm@unipanamericana.edu.co

Fecha de recepción: 04 de agosto de 2020 - Fecha de aceptación: 30 de septiembre de 2020

\begin{abstract}
Resumen
El presente artículo da cuenta de la implementación de una estrategia de apropiación social del conocimiento a través del semillero denominado STEAM +A el cual busca aportar al desarrollo social y económico del país a través de la promoción de vocaciones en ciencia, tecnología, ingeniería, artes y matemáticas en educación media articulada con educación superior. La experiencia adquirida ha generado la necesidad de validar, a través de un estudio observacional, la relación existente entre la percepción frente a las disciplinas STEAM +A y el género. En la recolección de información se implementó una encuesta de percepción que fue implementada en un periodo de dos años con un total de cinco mediciones. Debido a la fluctuación de los estudiantes en el servicio de educación pública de la ciudad de Bogotá D.C. (Colombia) la muestra presentó algunas modificaciones por lo que no se puede basar solo en los datos numéricos, sino que se requiere de información cualitativa para lograr una mayor comprensión de lo que sucede. Los resultados indican que no existe una asociación significativa entre el género y las vocaciones en las disciplinas anteriormente mencionadas.
\end{abstract}

Palabras claves: STEAM + A; formación interdisciplinar; vocaciones por género.

\begin{abstract}
This article reports the experience of a social appropriation of science strategy through a seedbed called STEAM +A which seeks to contribute to the social and economic development of the country through the promotion of vocations in science, technology, engineering, arts and mathematics in university and secondary education. The experience gained through the implementation of this strategy generated the need to validate through a study observational the relevance perceived by the female and male gender in relation to the disciplines. In the collection of information, a perception survey was used that was implemented over a period of two years to
\end{abstract}


young people who participated in the STEAM +A nursery. A total of five measurements were made and the sample varied due to the fluctuation of students at the secondary education level in a public school in the city of Bogotá D.C. (Colombia) it is complemented quantitatively with qualitative data, since they allow for a broader understanding of the situation analyzed. As results, it was identified that there is no significant association between gender and vocations in the disciplines associated with STEAM +A projects.

Keywords: STEAM + A; interdisciplinary training; vocations by gender.

\section{Introducción}

El comprender los retos que impone la sociedad actual exige que el sector educativo se adapte y se dé lugar a transformaciones que trasciendan más allá de la simple trasmisión de información al interior del aula. Desde el sector de la educación se tiene una gran responsabilidad con la sociedad en términos de lograr que el ser humano se forme integralmente impactando el desarrollo humano, social y económico de las regiones, lo cual redunda en el mejoramiento de la calidad de vida de las personas.

En este sentido, la simple transmisión de información en el aula debe ser reevaluada y llevarla a un nivel donde se convierta en conocimiento a través de tres elementos clave: motivación, tiempo y oportunidad de práctica (Cortés, 2020) cuando se aplica y se le da sentido, promoviendo de esta manera el desarrollo de la capacidad creativa para resolver problemas complejos y anticiparse a oportunidades futuras.

Desde el año 2017 el semillero STEAM + A se lleva a cabo en el Colegio Restrepo Millán de la ciudad de Bogotá D.C. y ha logrado una articulación interesante con la educación superior a través del Fundación Universitaria Panamericana a través de la facultad de ingeniería. Este semillero es visto como una estrategia de apropiación social del conocimiento, que ha favorecido el surgimiento de interrogantes tales como: ¿Cómo articular conocimientos y habilidades asociadas con el sector de la Tecnologías de la Información (TI) con problemas y necesidades del entorno? ¿Cuáles estrategias metodológicas permiten desarrollar competencias en los jóvenes para impactar su calidad de vida en el futuro? ¿Cómo fortalecer el desarrollo de competencias STEAM +A en jóvenes de educación media a través de ejercicios de trabajo colaborativo con estudiantes de formación superior? ¿Cómo establecer alianzas de cooperación entre la educación media y superior para promover las vocaciones asociadas a las disciplinas anteriormente mencionadas?

Los anteriores interrogantes se han ido esclareciendo con el pasar del tiempo, como resultado de la experiencia, pero hay un tema en particular que llama la atención y es la marcada diferencia que existe entre quienes se dedican a las disciplinas STEM en términos de género, tal es el punto que existen políticas públicas que promueven el acceso de la mujer en el campo científico, puesto que la diferencia es muy marcada. Este mismo fenómeno se presenta en el aula regular de educación media, el número de hombres que acceden a programas como el de Cisco, enfocado en el desarrollo de habilidades en el sector TI es notoriamente mayor al número de mujeres que acceden al mismo y es allí donde surge la pregunta que moviliza este documento ¿Cuál es la percepción que se tiene sobre la relevancia de disciplinas STEAM +A por parte de hombres y mujeres que participan en el Semillero? 


\section{Marco teórico}

El acrónimo STEM surge en Estados Unidos hacia el año 1990 como consecuencia de la carencia de estudiantes, de profesores y de profesionales en el sector y el surgimiento de iniciativas de formación interdisciplinar como una reforma educativa que tendría como principal objetivo introducir una metodología STEM en la educación secundaria que permitiera desarrollar a los estudiantes ciertas capacidades, como: a) El desarrollo de conocimientos y habilidades para identificar problemas en la vida cotidiana y la capacidad de obtener conclusiones basadas en evidencias, b) Comprensión de las características básicas de las disciplinas STEM, como una parte más del conocimiento humano, c) Concienciación sobre como las disciplinas STEM afectan a nuestro entorno material, intelectual y cultural, d) Disposición a participar en cuestiones del sector STEM con la intención de reducir la escasez de estudiantes en estudios superiores y en el sector laboral (Dolores et al., 2017, p. 6).

De acuerdo con los indicadores de la OCDE los títulos universitarios menos populares son en las áreas ciencias naturales, estadísticas y matemáticas con un 5\%; TIC con un 4\%; e ingeniería con un 17\% (OCDE, 2017). Esta cifra se disminuye en países como Colombia donde para el año 2018 solo el $15 \%$ de las se graduaron de la educación superior en 2018 optaron por un programa afín a la ciencia y la tecnología de acuerdo con los datos registrados en sistema SNIES del Ministerio de Educación Nacional. La Asociación Colombiana de Facultades de Ingeniería ACOFI ha puesto de manifiesto la preocupante situación de deserción en los primeros semestres de educación superior asociado principalmente con las carencias en el desarrollo de competencias básicas en las áreas de física, matemática y química (Velasquez, 2016).

Como se aprecia, los proyectos o programas STEM no son nuevos, sin embargo, en algunas instituciones educativas de la ciudad de Bogotá D.C. (Col) existe desconocimiento frente a las potencialidades de este tipo de iniciativas, debido principalmente, a la fragmentación del currículo y el conocimiento en disciplina, lo que llevada a una comprensión parcelada de la realidad; parece que se aprende matemáticas, física y química para pasar un examen, porque en el momento de hacer uso de esos conocimientos en la solución de problemas reales los jóvenes se paralizan. Países como Estados Unidos, Irlanda, México, España y algunos países de Latinoamérica han avanzado en la incorporación de este tipo de iniciativas (Cortés y Romero, 2018) . Es así, como dentro de los logros más destacados se identifica : a) el incremento de la participación de jóvenes en programas de ingeniería, ciencia y robótica, integrando estas disciplinas con el emprendimiento, b) el despertar vocaciones STEAM +A desde edad preescolar, c) la incorporación de las artes a través de la caricatura para promover la resolución de problemas usando la imaginación y habilidades matemáticas, d) el desarrollo del pensamiento crítico, habilidades de comunicación asertiva y sentido de pertenencia por la institución, e) la integración de instituciones de diversos puntos geográficos, formando comunidades a nivel local e internacional.

El Marco de Acción de Dakar Educación para Todos (EPT) plantea como compromiso el mejoramiento en la calidad del aprendizaje y la adquisición de valores humanos y competencias brindando acceso a técnicas y conocimientos necesarios para encontrar empleo remunerado y participar plenamente en la sociedad (Unesco, 2000). Estos programas que buscan mediante un acercamiento pedagógico y participativo fomentar la apropiación del conocimiento, no solo logran el mejoramiento de la calidad de vida y el desarrollo personal y profesional de los 
docentes, sino que impactan directamente el desarrollo de habilidades en los jóvenes inmersos en el sistema educativo (Cortés, 2018).

La presente experiencia pedagógica se ha estructurado en tres fases: i) transferencia de información y desarrollo de competencias técnicas específicas del sector TI, b) desarrollo de vocaciones STEAM +A, c) establecimiento de alianzas con instituciones externas.

La fase asociada a la transferencia de información y desarrollo de competencias técnicas específicas del sector TI se apoya en la plataforma de aprendizaje electrónico Cisco.netacad.com y se orienta a la consecución de certificaciones internacionales del sector TI. Las temáticas especificas se orientan desde un e-book con apoyo de simuladores virtuales como Packet Tracer y video juegos como Cisco Aspire, los cursos abordados son: Introducción al IoT, Fundamentos de IoT: conectando cosas, IT Essentials, para su desarrollo se apoya la programación con Arduino, el uso de máquinas virtuales como Virtual Box, introducción a Phyton y prácticas especificas asociadas a la manipulación de software y hardware.

Debido a que la información exigida en las certificaciones puede llegar a ser muy especializada para los jóvenes de colegios oficiales, impactando negativamente la permanencia, se generó una segunda fase donde el foco se trasladó al desarrollo de vocaciones STEAM +A, sin descuidar los contenidos propios de la plataforma pero agregando valor en términos de impacto en el gusto por la tecnología; esta transformación permitió que el estudiante comprendiera la transversalidad del currículo y desarrollara pensamiento computacional junto con habilidades blandas dando prioridad al trabajo en equipo, el trabajo por proyectos, la comunicación asertiva, la indagación de nuevas fuentes de consulta, la autogestión y la autorregulación entre otras. En esta fase, la principal herramienta metodológica ha sido la implementación del pensamiento de diseño inspirado en el Proyecto Design For Change (DFC).

La metodología de pensamiento de diseño se materializa a partir de cuatro momentos: siente, imagina, actúa y evalúa.

En una tercera fase, se logró el establecimiento de alianzas con instituciones externas en un trabajo mancomunado con una institución Universitaria y de esta forma, promover proyectos tecnológicos con impacto social. Desde el año 2009, se ha realizado el acercamiento a entidades externas y es así como a la fecha se cuenta con dos participaciones en Expo Ciencia y Expo tecnología (2009, 2017); de igual forma en el año 2017 el proyecto hizo parte activa del Programa Ondas de Colciencias, en el 2017 y la experiencia fue socializada a través del proyecto de comunidades de prácticas y saberes pedagógicos del IDEP. De igual forma, la práctica se ha articulado con trabajos de fin de carrera de estudiantes de ingeniería de telecomunicaciones, es así como en el año 2017, se desarrolló la implementación de un sistema de voz IP donde los estudiantes de secundaria participaban activamente en la administración de la solución; para el para el año 2018, se desarrolló una canasta IoT que busca promover la cultura del reciclaje y en el año 2019 se desarrolló una solución IoT para personas con distrofia muscular, en este ejercicio ha sido clave el establecimiento de alianzas con estudiantes y docentes.

Esto permite como estrategia de divulgación de la ciencia, acercar al joven a la vida universitaria y encontrar sentido a las competencias que desarrollan como resultado de la 
formación específica en el aula. Toda la documentación de estas iniciativas desarrolladas con programación en Arduino en la línea de IoT puede ser consultada en https://vomohifosi.jimdofree.com/

Los cambios generados en la práctica se desagregan a continuación como resultado de procesos de evaluación constante, investigación y observación de la práctica:

\section{Tabla 1}

\section{Cambios generados en la práctica}

\begin{tabular}{|c|c|c|}
\hline & $\begin{array}{l}\text { Problemáticas iniciales } \\
\text { identificada }\end{array}$ & Resultados obtenidos \\
\hline $\begin{array}{l}\text { Aprendizajes } \\
\text { de los } \\
\text { estudiantes }\end{array}$ & $\begin{array}{l}\text { Falencias en el desarrollo de } \\
\text { habilidades blandas y competencias } \\
\text { para el SS XXI, comprensión } \\
\text { lectora y técnica de estudio. } \\
\text { Formación para el trabajo: Las } \\
\text { posibilidades de avanzar en una } \\
\text { educación superior formal son } \\
\text { reducidas. Esto se convierte en una } \\
\text { limitante para el país en términos de } \\
\text { desarrollo y calidad de vida de sus } \\
\text { ciudadanos. }\end{array}$ & $\begin{array}{l}\text { Desarrollo de competencias blandas y para el ss XXI } \\
\text { basadas en la solución de problemas, la lectura crítica, } \\
\text { capacidades de indagación y aprender a aprender. } \\
\text { Estudiantes que ingresan a carreras del sector TI a } \\
\text { nivel profesional } \\
\text { Estudiantes que logran ubicarse laboralmente en el } \\
\text { sector TI } \\
\text { Estudiantes que han tenido la oportunidad de explorar } \\
\text { los contenidos propios de las certificaciones ofertadas } \\
\text { por la academia y que han decidido no incursionar en } \\
\text { este campo porque no corresponde a sus vocaciones, } \\
\text { esto impacta en indicadores de permanencia en } \\
\text { educación superior, muchas veces los jóvenes no saben } \\
\text { que estudiar e ingresan a carreras recomendadas por } \\
\text { sus padres. } \\
\text { Generación de tipo MOOC para la socialización del } \\
\text { proceso de desarrollo de las soluciones IoT }\end{array}$ \\
\hline $\begin{array}{c}\text { Procesos } \\
\text { institucionales }\end{array}$ & $\begin{array}{l}\text { Enseñanza tradicional basada en la } \\
\text { transmisión de información y } \\
\text { resistencia a otras formas de } \\
\text { aprender. }\end{array}$ & $\begin{array}{l}\text { Implementación de propuestas de formación basadas } \\
\text { en laboratorios, solución de retos y autoaprendizaje } \\
\text { Establecimiento de vínculos de colaboración con } \\
\text { docentes de la institución }\end{array}$ \\
\hline $\begin{array}{l}\text { Entorno y } \\
\text { comunidad }\end{array}$ & $\begin{array}{l}\text { Falta de articulación entre la } \\
\text { escuela, la comunidad y las } \\
\text { instituciones de educación superior. } \\
\text { Necesidad de impulsar el desarrollo } \\
\text { económico y social del país. } \\
\text { Necesidad de potenciar el desarrollo } \\
\text { de proyectos tecnológicos con } \\
\text { impacto social. }\end{array}$ & $\begin{array}{l}\text { Desarrollo de proyectos tecnológicos con impacto } \\
\text { social. Desarrollo de una solución IoT para personas } \\
\text { con distrofia muscular. Beneficiario: Estudiante de la } \\
\text { institución } \\
\text { Ampliación de oportunidades labores gracias a una } \\
\text { formación técnica especializada que es valorada a nivel } \\
\text { mundial. }\end{array}$ \\
\hline $\begin{array}{c}\text { Medio } \\
\text { ambiente }\end{array}$ & $\begin{array}{l}\text { Contenidos desarticulados de la } \\
\text { realidad }\end{array}$ & $\begin{array}{l}\text { Desarrollo de proyectos tecnológicos con impacto } \\
\text { social. Desarrollo de una solución IoT con impacto } \\
\text { ambiental }\end{array}$ \\
\hline
\end{tabular}

Fuente: Elaboración propia 
En la línea de programación y robótica con enfoque educativo STEM + A, se han articulado los conocimientos y la lógica de la programación con el desarrollo de competencias en electrónica para el desarrollo de soluciones IoT. Esto se realiza para dar respuesta a las necesidades del sector productivo y la sociedad en el término del desarrollo de ecosistemas inteligentes que impacten positivamente la vida de las personas y donde los jóvenes de las instituciones como futuros profesionales juegan un rol clave a nivel laboral y de emprendimiento.

Los estudiantes universitarios desarrollan prototipos funcionales y luego trasladan estos conocimientos a los jóvenes del colegio oficial a través de jornadas de socialización y difusión. En este escenario el esfuerzo por la búsqueda de aliados, el registro de la experiencia ha sido de gran ayuda el uso del blog y los grupos en Facebook como mecanismos de comunicación.

A continuación, se relacionan algunas de las investigaciones previas que dan origen a la presente propuesta a partir de sus temas de interés, ellos son: aprendizaje activo (Cortés y Cardona, 2013a)(Cortés y Parra, 2012), desarrollo profesional docente (Cortés et al., 2010a), diseño de escenarios de aprendizaje social (Cortés, 2016a), estrategias metodológicas (Cortés y Cardona, 2014a), innovación educativa (Cortés, 2016b, 2017a; A. Cortés y Cardona, 2013b), estrategias pedagógicas y calidad en programas de formación (Cortés y Cardona, 2014a) y políticas de incorporación de TIC en educación (Cortés, 2017b) entre otros (Cortés y Cardenas, 2010; Cortés, 2012; Cortés et al., 2010b, 2015, 2012; A. Cortés, 2014, 2016b, 2016c, 2017a, 2016d; Cortés y Cardona, 2015, 2013d, 2013a), Inteligencia Colectiva (Cortés, 2020) y finalmente, como resultado de la experiencia de estrategias de apropiación social de conocimiento a través de proyectos STEAM +A articulando educación media y superior (Cortés et al., 2019; Cortés y Romero, 2018).

\section{Metodología y alcance de la experiencia}

Este estudio se realiza en el marco de la estrategia de apropiación de conocimiento donde se realizó el seguimiento a un grupo de estudiantes adscritos al programa Cisco en el Colegio Restrepo Millán durante los años 2018 y 2019. La recolección de información se realizó a través de una encuesta de percepción que permite determinar la relevancia de las disciplinas STEAM +A asociadas con la variable género, y de esta manera determinar la asociación entre las variables a través de la prueba Chi-cuadrado de Pearson en cada uno de los casos.

Se trata de un estudio longitudinal con cinco medicaciones. El instrumento permitió recolectar la percepción de los participantes frente a: a) relevancia, aplicabilidad e interés por disciplinas STEAM +A, b) concepción sobre las personas dedicadas a la ciencia e interés por continuar en esta línea, c) interés por estudiar en un futuro d) interés de los padres frente al futuro profesional de sus hijos e) orientación profesional y f) interés por continuar estudios superiores.

La muestra corresponde a jóvenes entre los 15 y 18 años con la siguiente participación en términos de la edad: 15 años (33,1\%), 16 años $(29,8 \%), 17$ años $(28,9 \%)$, mayores de 17 años y menor de $14(8.2 \%)$. Se cuenta con un total de 184 respuestas generadas por el género femenino y 222 generadas por hombres. Al haberse realizado un total de cinco mediciones genera una participación promedio de 36 mujeres y 44 hombres para cada medición. 


\section{Resultados}

La hipótesis de trabajo es Ho = El género afecta la percepción que se tiene sobre la relevancia de $\mathrm{X}$, donde $\mathrm{X}$ representa la asignatura de análisis.

La escala de valoración utilizada es:

$\mathrm{B}=\mathrm{La}$ considero bastante relevante

$\mathrm{M}=\mathrm{La}$ considero medianamente relevante

$\mathrm{P}=\mathrm{La}$ considero poco relevante

$\mathrm{N}=$ La considero nada relevante

A continuación, se exponen los resultados obtenidos frente a la percepción que se tiene asociada a la relevancia de la biología:

\section{Tabla 2}

Percepción sobre la relevancia de la biología

\begin{tabular}{|c|c|c|c|c|c|c|c|c|c|c|}
\hline & \multicolumn{6}{|c|}{2018} & \multicolumn{4}{|c|}{2019} \\
\hline & \multicolumn{2}{|c|}{ FEB } & \multicolumn{2}{|c|}{ JUN } & \multicolumn{2}{|c|}{ NOV } & \multicolumn{2}{|c|}{ FEB } & \multicolumn{2}{|c|}{ JUN } \\
\hline & $\mathrm{H}$ & M & $\mathrm{H}$ & M & $\mathrm{H}$ & M & $\mathrm{H}$ & M & $\mathrm{H}$ & M \\
\hline B & 2 & 6 & 8 & 20 & 5 & 18 & 21 & 12 & 16 & 8 \\
\hline M & 3 & 4 & 19 & 22 & 15 & 5 & 33 & 23 & 28 & 14 \\
\hline $\mathrm{P}$ & 1 & 1 & 10 & 4 & 5 & 0 & 20 & 12 & 10 & 6 \\
\hline $\mathrm{N}$ & 2 & 3 & 3 & 2 & 8 & 0 & 7 & 2 & 2 & 1 \\
\hline $\mathrm{n}$ & 22 & & 88 & & 56 & & 130 & & 85 & \\
\hline $\mathrm{P}$ & 0.8582 & & 0.058 & & $2.1 \times 10^{-5}$ & & 0.74 & & 0.992 & \\
\hline
\end{tabular}

$*_{\mathrm{n}}=$ tamaño de la muestra

$* \mathrm{p}=$ Chi cuadrado

Fuente: Elaboración propia

Como se puede apreciar en la tabla solo para el año 2018 en el mes de noviembre el género fue significativo en términos de la relevancia que tiene la biología para los jóvenes pertenecientes al proyecto. Por lo tanto, se concluye que el género no afecta la percepción que se tiene sobre la biología.

A continuación, se exponen los resultados obtenidos frente a la percepción que se tiene asociada a la relevancia de la física y la química: 


\section{Tabla 3}

Percepción sobre la relevancia de la física y la química

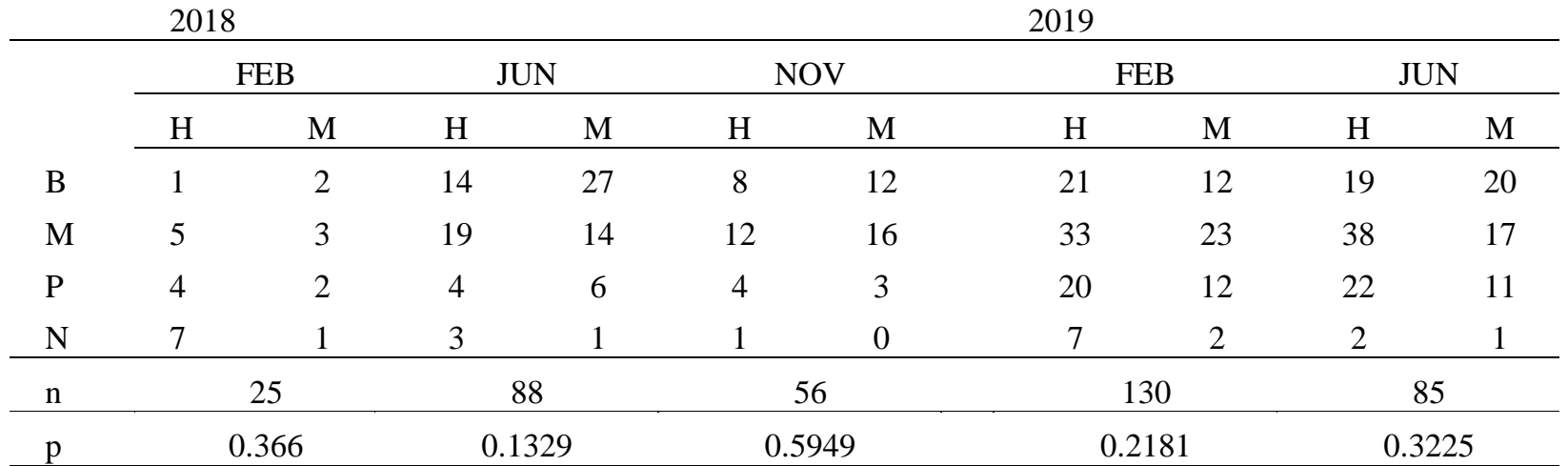

$*_{\mathrm{n}}=$ tamaño de la muestra

$* \mathrm{p}=$ Chi cuadrado

Fuente: Elaboración propia

Se concluye que el género no afecta la percepción que se tiene sobre la física y la química.

A continuación, se exponen los resultados obtenidos frente a la percepción que se tiene asociada a la relevancia de la tecnología:

\section{Tabla 4}

Percepción sobre la relevancia de la tecnología

\begin{tabular}{|c|c|c|c|c|c|c|c|c|c|c|}
\hline & \multicolumn{4}{|c|}{2018} & \multicolumn{6}{|c|}{2019} \\
\hline & \multicolumn{2}{|c|}{ FEB } & \multicolumn{2}{|c|}{ JUN } & \multicolumn{2}{|c|}{ NOV } & \multicolumn{2}{|c|}{ FEB } & \multicolumn{2}{|c|}{ JUN } \\
\hline & $\mathrm{H}$ & $\mathrm{M}$ & $\mathrm{H}$ & $\mathrm{M}$ & $\mathrm{H}$ & $\mathrm{M}$ & $\mathrm{H}$ & $\mathrm{M}$ & $\mathrm{H}$ & $\mathrm{M}$ \\
\hline B & 4 & 2 & 27 & 24 & 15 & 14 & 51 & 25 & 31 & 14 \\
\hline M & 2 & 4 & 8 & 17 & 9 & 4 & 25 & 19 & 19 & 8 \\
\hline $\mathrm{P}$ & 9 & 11 & 4 & 6 & 1 & 1 & 2 & 4 & 4 & 6 \\
\hline $\mathrm{N}$ & 4 & 8 & 1 & 1 & 12 & 0 & 3 & 1 & 2 & 1 \\
\hline $\mathrm{n}$ & \multicolumn{2}{|c|}{44} & \multicolumn{2}{|c|}{88} & \multicolumn{2}{|c|}{56} & \multicolumn{2}{|c|}{130} & \multicolumn{2}{|c|}{85} \\
\hline $\mathrm{p}$ & \multicolumn{2}{|c|}{0.5545} & \multicolumn{2}{|c|}{0.3742} & \multicolumn{2}{|c|}{0.02782} & \multicolumn{2}{|c|}{0.2923} & \multicolumn{2}{|c|}{0.3334} \\
\hline
\end{tabular}

$*_{\mathrm{n}}=$ tamaño de la muestra

$* \mathrm{p}=$ Chi cuadrado

Fuente: Elaboración propia

Se concluye que el género no afecta la percepción que se tiene sobre la tecnología. 
A continuación, se exponen los resultados obtenidos frente a la percepción que se tiene asociada a la relevancia de las matemáticas:

\section{Tabla 5}

Percepción sobre la relevancia de las matemáticas

\begin{tabular}{|c|c|c|c|c|c|c|c|c|c|c|}
\hline & \multicolumn{4}{|l|}{2018} & \multicolumn{6}{|c|}{2019} \\
\hline & \multicolumn{2}{|c|}{ FEB } & \multicolumn{2}{|c|}{ JUN } & \multicolumn{2}{|c|}{ NOV } & \multicolumn{2}{|c|}{ FEB } & \multicolumn{2}{|c|}{ JUN } \\
\hline & $\mathrm{H}$ & M & $\mathrm{H}$ & M & $\mathrm{H}$ & $\mathrm{M}$ & $\mathrm{H}$ & $\mathrm{M}$ & $\mathrm{H}$ & M \\
\hline B & 4 & 3 & 24 & 18 & 18 & 18 & 44 & 30 & 31 & 24 \\
\hline M & 2 & 1 & 10 & 5 & 6 & 1 & 29 & 13 & 17 & 4 \\
\hline $\mathrm{P}$ & 7 & 5 & 6 & 1 & 1 & 2 & 4 & 4 & 5 & 1 \\
\hline $\mathrm{N}$ & 2 & 1 & 24 & 0 & 10 & 0 & 4 & 2 & 3 & 0 \\
\hline $\mathrm{n}$ & \multicolumn{2}{|c|}{25} & \multicolumn{2}{|c|}{88} & \multicolumn{2}{|c|}{56} & \multicolumn{2}{|c|}{130} & \multicolumn{2}{|c|}{85} \\
\hline $\mathrm{p}$ & \multicolumn{2}{|c|}{0.9854} & \multicolumn{2}{|c|}{0.001803} & \multicolumn{2}{|c|}{0.01121} & \multicolumn{2}{|c|}{0.652} & \multicolumn{2}{|c|}{0.000285} \\
\hline
\end{tabular}

$*_{\mathrm{n}}=$ tamaño de la muestra

$* \mathrm{p}=$ Chi cuadrado

Fuente: Elaboración propia

En la encuesta realizada en los meses de junio y noviembre de 2018, lo mismo que en junio de 2019 concluyen que el género afecta la percepción que se tiene de la relevancia de las matemáticas. Para los otros periodos se concluye que el género no afecta la percepción.

A continuación, se exponen los resultados obtenidos frente a la percepción que se tiene asociada a la relevancia de las artes:

Tabla 6

Percepción sobre la relevancia de las artes

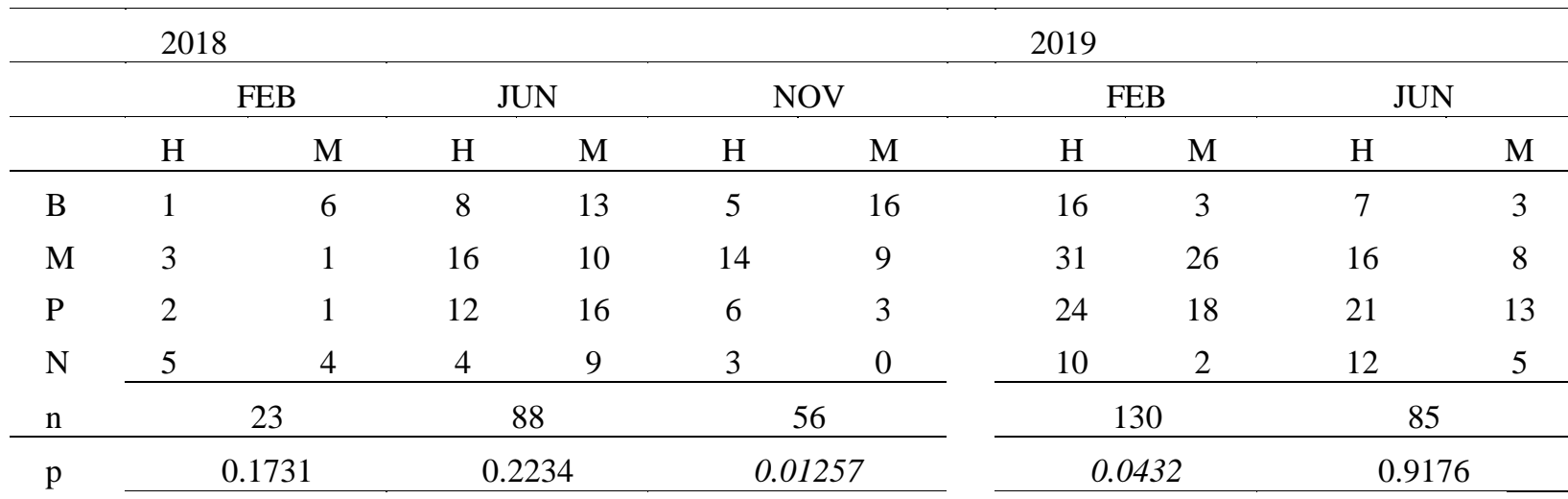

*n $=$ tamaño de la muestra

$* \mathrm{p}=$ Chi cuadrado

Fuente: Elaboración propia 
En noviembre de 2018 con $\mathrm{p}=0.01257$ se determina que el género afecta la percepción que se tiene sobre las artes, lo mismo se observa en el mes de febrero de 2019. Para las otras medicaciones se concluye que el género no afecta la percepción que se tiene sobre las artes.

A continuación, se exponen los resultados obtenidos frente a la percepción que se tiene asociada a la relevancia de la ingeniería:

\section{Tabla 7}

Percepción sobre la relevancia de la ingeniería

\begin{tabular}{|c|c|c|c|c|c|c|c|c|c|}
\hline & \multicolumn{5}{|c|}{2018} & & \multicolumn{3}{|c|}{2019} \\
\hline & FEB & & JUN & & NOV & & FEB & & JUN \\
\hline & $\mathrm{H}$ & M & $\mathrm{H}$ & $\mathrm{M}$ & $\mathrm{H}$ & $\mathrm{M}$ & $\mathrm{H}$ & $\mathrm{M}$ & $\mathrm{H}$ \\
\hline B & 4 & 2 & 22 & 14 & 13 & 6 & 37 & 19 & 23 \\
\hline M & 2 & 1 & 12 & 25 & 9 & 20 & 26 & 16 & 19 \\
\hline $\mathrm{P}$ & 5 & 4 & 5 & 9 & 2 & 5 & 14 & 8 & 10 \\
\hline $\mathrm{N}$ & 6 & 1 & 1 & 0 & 1 & 0 & 4 & 6 & 4 \\
\hline $\mathrm{n}$ & 25 & & 88 & & 56 & & 130 & & 85 \\
\hline $\mathrm{p}$ & 0.6465 & & 0.04976 & & 0.03687 & & 0.4796 & & 0.027 \\
\hline
\end{tabular}

*n = tamaño de la muestra

$* \mathrm{p}=$ Chi cuadrado

Fuente: Elaboración propia

En junio y noviembre de 2018, así como en junio de 2019 se determina que el género afecta la percepción que se tiene sobre la ingeniería. Para las otras mediciones se concluye que el género no afecta.

A continuación, se exponen los resultados obtenidos frente a la percepción que se tiene asociada a la relevancia de las ciencias:

\section{Tabla 8}

Percepción sobre la relevancia de las ciencias 2018

\begin{tabular}{lcccccc}
\hline & 2018 & & & & \\
\hline & & FEB & & JUN & NOV \\
\cline { 2 - 6 } & $\mathrm{M}$ & $\mathrm{H}$ & $\mathrm{M}$ & $\mathrm{H}$ & $\mathrm{M}$ & $\mathrm{H}$ \\
$\mathrm{B}$ & 3 & 1 & 7 & 7 & 18 & 9 \\
$\mathrm{M}$ & 13 & 3 & 2 & 22 & 23 & 13 \\
$\mathrm{P}$ & 8 & 2 & 3 & 9 & 7 & 3 \\
$\mathrm{~N}$ & 5 & 4 & 2 & 2 & 0 & 5 \\
$\mathrm{n}$ & & 24 & & 88 & & 56 \\
$\mathrm{nyyyyy} \mathrm{p}$ & & 0.1689 & 0.09213 & & 0.001055 \\
\hline
\end{tabular}

Fuente: Elaboración propia 


\section{Tabla 9}

Percepción sobre la relevancia de las ciencias

\begin{tabular}{cccccc}
\hline & & FEB & JUN & \\
\hline & M & H & M & H & M \\
B & 21 & 20 & 13 & 6 & 7 \\
M & 5 & 36 & 25 & 34 & 15 \\
P & 0 & 20 & 8 & 14 & 6 \\
N & 0 & 5 & 3 & 85 & 1 \\
\hline $\mathrm{n}$ & & 130 & & 0.4452
\end{tabular}

Fuente: Elaboración propia

En noviembre de 2018 se determina que el género afecta la percepción que se tiene sobre las ciencias. Para las otras mediciones se concluye que el género no afecta la percepción.

Con el fin de complementar la anterior información, se indago en relación con otros ítems que se consideran relevantes frente a la percepción que los jóvenes tienen de las disciplinas STEAM +A. Los resultados obtenidos son:

1. Frente a la pregunta $¿$ Te parece llamativa la vida de una persona que se dedica a la ciencia, la tecnología o las matemáticas (en términos de estabilidad económica, laboral, posibles cargos laborales, etc)? Un $89.3 \%$ considera este tipo de vida como llamativa y al $10.7 \%$ no.

2. El $41.3 \%$ considera que disfrutaría una profesión o actividades relacionadas con la ingeniería o ser inventor; el $23.1 \%$ se inclina por ser deportista; un 27, 3\% se inclina por las artes; un $28 \%$ por ser empresario o directivo; un 31,4 ser médico y con una participación del $0.8 \%$ se encuentran áreas como veterinaria, chef, músico, mecánica y psicología. Cabe resaltar que se podía seleccionar más de un área de actuación.

3. Los jóvenes reconocen que un $62.8 \%$ de sus padres se encuentran interesados en que ellos avancen en procesos de formación dentro de la línea STEAM +A; el 21.5\% a música, artes y diseño; el 7.4\% a las letras y el restante a medicina, química, trabajo social, entre otros.

4. Un 44,6\% de los jóvenes manifiestan que ha recibido información suficiente de orientación vocacional, un $23,1 \%$ nada; el $26,4 \%$ considera que poca y el $5.8 \%$ considera que bastante.

5. Un $90.9 \%$ manifiesta su interés por continuar estudiando una vez culmine el colegio el $8.3 \%$ manifiesta que tal vez y el $0.8 \%$ afirma que no lo hará. Dentro de las razones para continuar o no, se encuentra: interés por saber más, crear su propia empresa, mejorar sus oportunidades, progreso, estabilidad laboral, porque le apasiona, por la situación económica, independencia económica. 


\section{Conclusiones}

El alcance del estudio se centra en identificar la dependencia que existente entre la relevancia de disciplinas STEAM + A ciencias (biología, física, química), tecnología, ingeniería, artes, matemáticas y su relación con el género de los participantes de una iniciativa de apropiación social de la ciencia denominada Semillero STEAM +A. A manera de conclusión:

1. En la medición 2018 (3) se determinan que el género es significativo en términos de la relevancia que tiene la biología para los jóvenes consultados. En las otras mediciones no es significativo.

2. En las cinco mediciones se determina que el género no afecta la percepción que se tiene sobre la física y la química.

3. En las cinco mediciones se determina que el género no afecta la percepción que se tiene sobre la tecnología.

4. En la medición 2018 (2-3), así como 2019 (2) se concluye que el género afecta la percepción que se tiene de la relevancia de las matemáticas.

5. En la medicación 2018 (2) se determina que el género afecta la percepción que se tiene sobre las artes, lo mismo se observa en 2019 (1). En las otras mediciones se concluye que el género no afecta la percepción que se tiene sobre las artes.

6. En las mediciones 2018 (2-3) así como en 2019 (2) se determina que el género afecta la percepción que se tiene sobre la ingeniería. Para las otras mediciones se concluye que el género no afecta la percepción que se tiene.

7. En la medición 2018 (2) se determina que el género afecta la percepción que se tiene sobre las ciencias, en las otras mediciones se concluye que el género no afecta la percepción.

Adicionalmente, el estudio permitió determinar:

Un $89.3 \%$ de los encuestados considera como llamativa la vida de una persona que se dedica a la ciencia, la tecnología o las matemáticas en términos de estabilidad económica, laboral, posibles cargos laborales, etc., este fenómeno permite conjeturar frente a la oportunidad de potenciar este tipo de formación al interior de las instituciones educativas y de esta manera favorecer el acceso a mejores oportunidades laborales para los jóvenes una vez culminen su formación media puesto que el $41.3 \%$ de los encuestados consideran que disfrutaría una profesión o actividades relacionadas con la ingeniería o ser inventor.

Por otro lado, desde la perspectiva de los jóvenes, ellos manifiestan que un $62.8 \%$ de sus padres que se encuentran interesados en que ellos avancen en programas de formación de la línea STEAM +A. En relación con la orientación vocacional, se manifiestan niveles aun preocupantes frente al acompañamiento de la institución educativa frente al tema, lo cual puede ser aprovechado para dar respuesta al $90.9 \%$ de los jóvenes que manifiestan su interés por continuar estudiando una vez culmine el colegio, ampliando oportunidades en término del acompañamiento y la orientación vocacional para facilitar el acceso y permanencia de los jóvenes en la educación superior. 


\section{Bibliografía}

Cortés, A, y Cardenas, M. (2010). Lineamientos pedagógicos para cursos en modalidad virtual.

Cortés, Albenis. (2012). Uso pedagógico de Herramientas Web 2 . 0 en la labor docente. Revista Iberoamericana Para La Investigación y El Desarrollo Educativo., 8.

Cortés, Albenis. (2014). Instrumento de autoevaluación de los programas de formación virtual a partir de criterios básicos de calidad. Revista Praxis, 10, 165-175. https://doi.org/http://dx.doi.org/10.21676/23897856.1367

Cortés, Albenis. (2016a). Diseño de escenarios de aprendizaje social: aportes teóricos para su construcción. In A. A. científica de psicología y Educación (Ed.), Psicología y educación: presente y futuro.

Cortés, Albenis. (2016b). Prácticas innovadoras de integración educativa de TIC que posibilitan el desarrollo profesional docente. Un estudio en Instituciones de niveles básica y media de la ciudad de Bogotá (Col). [Universidad Autónoma de Barcelona]. http://www.tdx.cat/handle/10803/400225\#.WL2TflmdcXw.linkedin

Cortés, Albenis. (2016c). Proyectos de innovación: Características de las instituciones donde se promueven prácticas de integración educativa de TIC. In CIPE (Ed.), Psicología y Educación : Presente y Futuro.

Cortés, Albenis. (2017a). Innovación y TIC en educación ¿ La incorporación educativa de las TIC es por sí sola innovación ? IOSR Journal of Research\&Method in Education, 7(4), 7-10. https://doi.org/10.9790/7388-0704030710

Cortés, Albenis. (2017b). Políticas públicas para la integración de las TIC en educación. 2017 Revista Educación Y Ciudad Del IDEP (pp. 33, 75-85).

Cortés, Albenis. (2018). Innovación educativa: convirtiendo conocimiento en nuevas oportunidades (Ediciones de la U (ed.)).

Cortés, Albenis. (2020). Inteligencia colectiva desde la gestión del conocimiento. In Albenis

Cortés (Ed.), Inteligencia colectiva desde la gestión del conocimiento (Ediciones).

Cortés, Albenis. (2016d). Diseño de escenarios de aprendizaje social: aportes teóricos para su construcción. In A. A. científica de psicología y Educación (Ed.), Psicología y educación: presente y futuro. https://doi.org/ISBN: 9788460887140

Cortés, Albenis, Aponte, C., Fonseca, A., y Trujillo, C. (2010b). La evaluación docente desde el ámbito administrativo. The Evaluation of Teaching from the Administrative Matter. Actualidades Pedagógicas, 56, 133-143.

Cortés, Albenis, y Cardona, M. (2013a). Elementos pedagógicos a considerar para la generación de un proceso de tutoría virtual eficiente 1 Albenis Cortés, Jenny Marcela Cardona. XIV Encuentro Internacional Virtual Educa Colombia. http://www.virtualeduca.info/ponencias2013/verponencias.php A.

Cortés, Albenis, y Cardona, M. (2013b). Identificación de nuevos caminos hacia la innovación educativa. Revista Iberoamericana Para La Investigación Y El Desarrollo Educativo.

Cortés, Albenis, y Cardona, M. (2013d). Intereses de los docentes que promueven la implementación de herramientas de la web 2.0. In Congreso Cirtual Educa 2013. Memorias del Congreso Internacional Virtual Educa 2013. http://www.virtualeduca.info/ponencias2013/verponencias.php

Cortés, Albenis, y Cardona, M. (2014b). Contribución de las simulaciones como estrategia metodológica. Revista Internacional de Evaluación y Medición de La Calidad Educativa, 1(Metodologías activas en educación), 19-30. https://doi.org/2386-7787 
Cortés, Albenis, y Cardona, M. (2015). Evaluación de los programas de formación virtual por parte de tutores.

Cortés, Albenis, y Cardona, M. (2013e). La web 2 . 0 en función de los intereses docentes : Identificación de nuevos caminos hacia la innovación educativa. In C. A.C. (Ed.), Congreso Virtual Internacional sobre educación media y superior. Centro de estudios e investigación para el desarrollo docente.

Cortés, Albenis, Cardona, M., y Parra, J. (2012). Impacto de la implementación de material digital y virtual como apoyo a la educación presencial. In SOCOTE (Ed.), IV Congreso Iberoamericano de Soporte al Conocimiento con la Tecnología SOCOTE.

Cortés, Albenis, Jaramillo, I., y Cardenas, M. (2015). Guía de la comunidad Gurugle para el diseño de nodos de conocimiento. www.gurugle.com

Cortés, Albenis, y Parra, J. (2012). Aprendizaje activo. Documento de trabajo.

Cortés, Albenis, y Romero, D. (2018). Semillero STEAM: Una propuesta de articulación entre la educación media y superior. In Editorial Instituto Antioqueño de Investigación (Ed.), Investigación formativa en ingeniería (pp. 259-264).

Cortés, Albenis, Villalobos, W., Lemus, J., Rodríguez, D., y Vargas, A. (2019). Solución Internet de las cosas para personas con movilidad reducida. In Editorial Instituto Antioqueño de Investigación (Ed.), Investigación formativa en ingeniería (pp. 40-51).

Dolores, M., Celdrán, J., Olga, D. :, y Arnaldo, R. (2017). Análisis de la situación de la mujer en el sector STEM. Comparativa España-Países Bajos.

OCDE. (2017). Panorama de la educación: Indicadores de la OCDE. https://www.oecdilibrary.org/education/panorama-de-la-educacion-2017_eag-2017-es

Unesco. (2000). Foro mundial sobre la educación. Marco de Acción de Dakar. Marco de Acción de Dakar. http://unesdoc.unesco.org/images/0012/001211/121147s.pdf

Velasquez, L. (2016). En Colombia se necesitan 18.000 ingenieros más. https://www.elcolombiano.com/colombia/educacion/en-colombia-se-necesitan-18-000ingenieros-mas-JM3970856 